\title{
Investigation of Oxide Phases of MoS2: van der Waals Epitaxially Formed $\alpha$-MoO3 on MoS2
}

\section{Aram Yoon and Zonghoon Lee}

Center for Multidimensional Carbon Materials, Institute for Basic Science (IBS), Ulsan 44919, Republic of Korea, United States

Two-dimensional (2D) transition metal dichalcogenides (TMDs), e.g., $\mathrm{MoS}_{2}, \mathrm{WS}_{2}$, and $\mathrm{WSe}_{2}$, have received great interests because of their unique electrical, optical, and mechanical properties. Oxidation of TMDs is known to significantly influence their properties. Hence, tuning the properties of TMDs through oxidation has been actively studied. [1] Especially, heterostructure of TMDs and transition metal oxides (TMOs) have potential to be applied as a p-type semiconductor, because TMO is a promising candidate for a hole injection layer. However, investigation of oxidation behavior of TMDs is challenging because the amount of TMOs formed from the oxidation is small and they are structurally instable. Especially, studying oxidation of $\mathrm{MoS}_{2}$ is difficult because that $\mathrm{MoO}_{3}$ are soluble in water and easily damaged by beam irradiation. Here, we analyzed the oxidation behavior of $2 \mathrm{D} \mathrm{MoS}_{2}$ focusing on their morphologies and phases. The analysis was performed using aberration-corrected TEM (Titan3 G2 60300, FEI). TEM and STEM images were taken under $200 \mathrm{kV}$ acceleration voltage. Electron energy loss spectroscopy (EELS) was obtained using a Gatan Quantum 965 dual EELS system under $80 \mathrm{kV}$ acceleration voltage. The energy spread of the monochromated zero-loss peak was $0.15 \mathrm{eV}$ in full width at half-maximum with an energy dispersion of $0.01 \mathrm{eV} / \mathrm{ch}$. [2] 2D $\mathrm{MoS}_{2}$ begins to oxidize at approximately $400{ }^{\circ} \mathrm{C}$ from the edges of the flakes. The oxides are orthorhombic phase molybdenum trioxide $\left(\alpha-\mathrm{MoO}_{3}\right)$ which have layered structure. The $\alpha-\mathrm{MoO}_{3}$ nanosheets are stacked on the $\mathrm{MoS}_{2}$ with a van der Waals interaction. In addition, the nanosheets are generated epitaxially in six orientations. (Figure 1) Band gap and stoichiometry of the oxides are measured using EELS. We found that the band gap of $\mathrm{MoS}_{2}$ is modified from 1.27 to $3.0 \mathrm{eV}$ through thermal oxidation. Moreover, stoichiometry changes are not observed according to its position [3]. This study can be applied to most of TMDs and offer new strategies apply TMD-TMO heterostructure in various applications as p-type transistor, gas sensors, and photocatalysts. [4]


Figure 1. (a) BF and (b,c) DF-TEM image of $\mathrm{MoS}_{2}$ annealed in air at $400{ }^{\circ} \mathrm{C}$ for $1 \mathrm{~h}$. DF-TEM images of (b) $\mathrm{MoS}_{2}$ obtained from (110) diffraction spots of $\mathrm{MoS}_{2}$ and (c) $\mathrm{MoO}_{3}$ obtained from (002) diffraction spots of $\mathrm{MoO}_{3}$. Atomic-resolution STEM images of (d) $\mathrm{MoS}_{2}$, (e) $\mathrm{MoO}_{3} / \mathrm{MoS}_{2}$ heterostructure, and (f) MoO3. In FFT patterns, $\mathrm{MoS}_{2}$ and $\mathrm{MoO}_{3}$ are marked as yellow and red, respectively. The scale bars of (a-c) and (d-h) are 500 and $2 \mathrm{~nm}$, respectively. (g) Zero-loss subtracted low-loss EEL spectra of pristine, 
200, 300, and $400{ }^{\circ} \mathrm{C}$-annealed $\mathrm{MoS}_{2}$ and bulk $\mathrm{MoO}_{3}$. (h) Magnified low-loss EEL spectra of pristine and $400{ }^{\circ} \mathrm{C}$-annealed MoS2. The result was compared with bulk $\mathrm{MoO}_{3}$ to determine the change in $\mathrm{MoS}_{2}$ band gap after oxidation. Reprinted from [2] with permission of American Chemical Society.

(a)



(b)
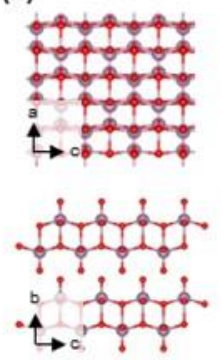

(c)



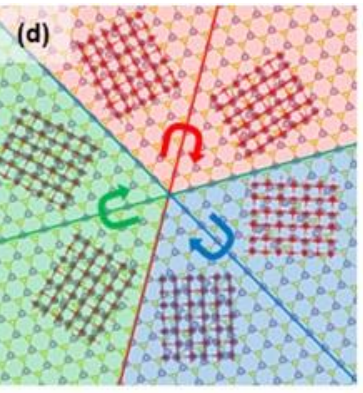

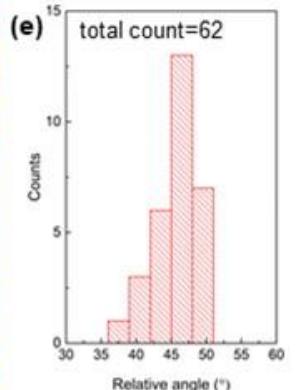

Figure 2. Figure 2. Crystal structure of (c) $2 \mathrm{H}-\mathrm{MoS}_{2}$ and (d) $\alpha-\mathrm{MoO}_{3}$. (c) SAED pattern and (d) Schematic diagram of the heterostructure of $\alpha-\mathrm{MoO}_{3}$ and $\mathrm{MoS}_{2}$. (e) Histogram of relative angles measured by peaks of $\mathrm{MoS}_{2}$ and $\mathrm{MoO}_{3}$ shows that $\alpha-\mathrm{MoO}_{3}$ nanosheets are van der Waals epitaxially formed on $\mathrm{MoS}_{2}$. Reprinted from [2] with permission of American Chemical Society.

References

[1] J. Jadwiszczak et al., Sci. Adv., 4.3 (2018), eaao5031

[2] A. Yoon et al., ACS Appl. Mater. Interfaces 12. 19 (2020), p. 22029-22036

[3] L. Lajaunie et al., Ultramicroscopy 149 (2015), p. 1-8.

[4] Work supported by NRF (2018R1A2A2A05019598) and IBS (IBS-R019-D1). 